\title{
Análise comparativa entre técnicas morfométricas aplicadas a Odontesthes bonariensis (Valenciennes) e Odontesthes humensis De Buen (Osteichthyes, Atherinopsidae)
}

\author{
Marlise de Azevedo Bemvenuti ${ }^{1,2}$ \\ Fábio Lameiro Rodrigues ${ }^{1,3}$
}

\begin{abstract}
Comparative analysis among two different morphometric techniques used in Odontesthes bonariensis and Odontesthes humensis (Atherinopsidae). The objective of this study was to compare two different morphometric methodologies - traditional measurements and truss networks - based on two species of silversides, Odontesthes bonariensis (Valenciennes, 1835) and O. humensis De Buen, 1953, to determine which of the methods results in more reliable distinction between groups. The traditional measures do not represent the true fish form because it does not take into account alometric variations or distinct growth stages. The measures with truss networks extend over the fish form thoroughly resulting in complete representation of the fish's body when homologous morphologic points are connected. The two sets of measures used in the principal components analysis resulted in two groups. The first principal component presented similar positive coefficients, being interpreted as a variation of "size", due to different stages of fish growth. The second component presented positive and negative coefficients, with different values that were interpreted as changes in the "shape" of the organisms. As the groups overlapped partially on the second component axis (the one that represents shape), data were adjusted to exclude the size effect. The first component, then, presented positive and negative coefficients that were related to changes in the shape of organisms. The variables with positive coefficients were related to the anterior area of the body, while the negative coefficients were associated with the caudal peduncle. These variables were the same ones found in both the adjusted and non-adjusted analysis. This study showed that measures with truss networks represented more clearly the difference between groups of $O$. bonariensis and $O$. humensis, than with the traditional measures.

KEY WORDS. Atherinopsidae, multivariate morphometry, truss networks, silverside
\end{abstract}

Estudos da forma do corpo em relação ao tamanho por métodos numéricos são de grande importância na área da biologia evolutiva (REYMENT et al. 1984; REIS 1988; ROHLF 1990). O desenvolvimento de métodos de análise multivariada e a disponibilidade de pacotes estatísticos tem permitido uma avaliação da variação integrada do organismo e comparações de formas e estruturas, necessárias ao estudo da sistemática (NEFF \& MARCUS 1980; REYMENT 1985; REIS 1988; ROHLF 1990).

1) Departamento de Oceanografia, Fundação Universidade Federal do Rio Grande. Caixa Postal 474, 96201-900 Rio Grande, Rio Grande do Sul, Brasil.

2) E-mail: docmab@furg.br

3) E-mail: oceaflr@ furg.br

Revta bras. Zool. 19 (3): 789 - 796, 2002 
Os principais problemas enfrentados pela morfometria têm sido a medição do grau de similaridade entre duas ou mais formas, a determinação de variáveis para serem analisadas e a transformação destas variáveis em estimativas de diferenças na forma dos organismos (REIS 1988). Segundo BOOKSTEIN (1993), a confusão na morfometria aconteceu porque não havia consenso sobre quais seriam os dados morfométricos apropriados.

Caracteres morfométricos obtidos por medição tradicional não levam em conta a variação alométrica ou os diferentes estágios de crescimento, o que ocorre naturalmente nos indivíduos. Conseqüentemente, estes dados tendem a provocar erros de análise, sendo difíceis de interpretar, além de não representarem a realidade da forma dos organismos.

Recentemente, técnicas de morfometria multivariada têm selecionado caracteres que representam o corpo como um todo, como é o caso das redes de treliça, que utilizam pequenas distâncias medidas, conectando pontos anatômicos, homólogos entre os indivíduos, permitindo sua comparação (HUMPHRIES et al. 1981; STRAUSS \& BOOKSTEIN 1982). As diferenças de forma são detectadas em todas as direções, compensando erros aleatórios de medição. Além de descrever os padrões de crescimento e alometria dentro das populações, podem discriminar grupos de organismos que variam em forma e tamanho (STRAUSS \& BOOKSTEIN 1982).

O objetivo deste trabalho foi comparar as metodologias baseadas em medidas tradicionais com as em redes de treliça para distinção entre duas espécies de peixes-rei, Odontesthes bonariensis (Valenciennes, 1835) e Odontesthes humensis De Buen, 1953.

\section{MATERIAL E MÉTODOS}

Foram obtidas duas séries de variáveis, medidas nos mesmos exemplares de Odontesthes bonariensis $(\mathrm{n}=26)$ e Odontesthes humensis $(\mathrm{n}=25)$, capturados nas lagoas costeiras do Estado do Rio Grande do Sul e depositados na Coleção de Peixes, Fundação Universidade Federal do Rio Grande.

$\mathrm{Na}$ série de medidas tradicionais foram obtidas 25 variáveis detalhadas em BEMVENUTI (2002): comprimento padrão (cp); distância do focinho até cada nadadeira (pd1, pd2, pan, pp1, pp2); comprimento da cabeça (cab); altura máxima da cabeça (altcab); focinho (foc); diâmetro horizontal do olho (olho); distância interorbital (intorb); largura da boca (boca); comprimento da maxila (max); altura mínima do pedúnculo caudal (altped); distância entre as origens da peitoral e pélvica (p1p2); distância entre as nadadeiras e o pedúnculo caudal (anpc, d1pc, d2pc, p1pc, p2pc); distância entre as origens das dorsais (d1d2); distância entre as origens da anal e demais nadadeiras (and1, and2, anp1, anp2) (Fig. 1).

$\mathrm{Na}$ série de medidas da rede de treliça, de acordo com STRAUSS \& BOOKSTEIN (1982), foram obtidas 21 variáveis a partir dos 10 marcos anatômicos selecionados: 1) margem anterior do focinho; 2) extremidade posterior da maxila; 3) extremidade posterior da caixa craniana; 4) base posterior do opérculo; 5) base da primeira nadadeira dorsal; 6) base da nadadeira pélvica; 7) base posterior da segunda nadadeira dorsal; 8) base da nadadeira anal; 9) extremidade superior do pedúnculo caudal; 10) extremidade inferior do pedúnculo caudal (Fig. 2). 

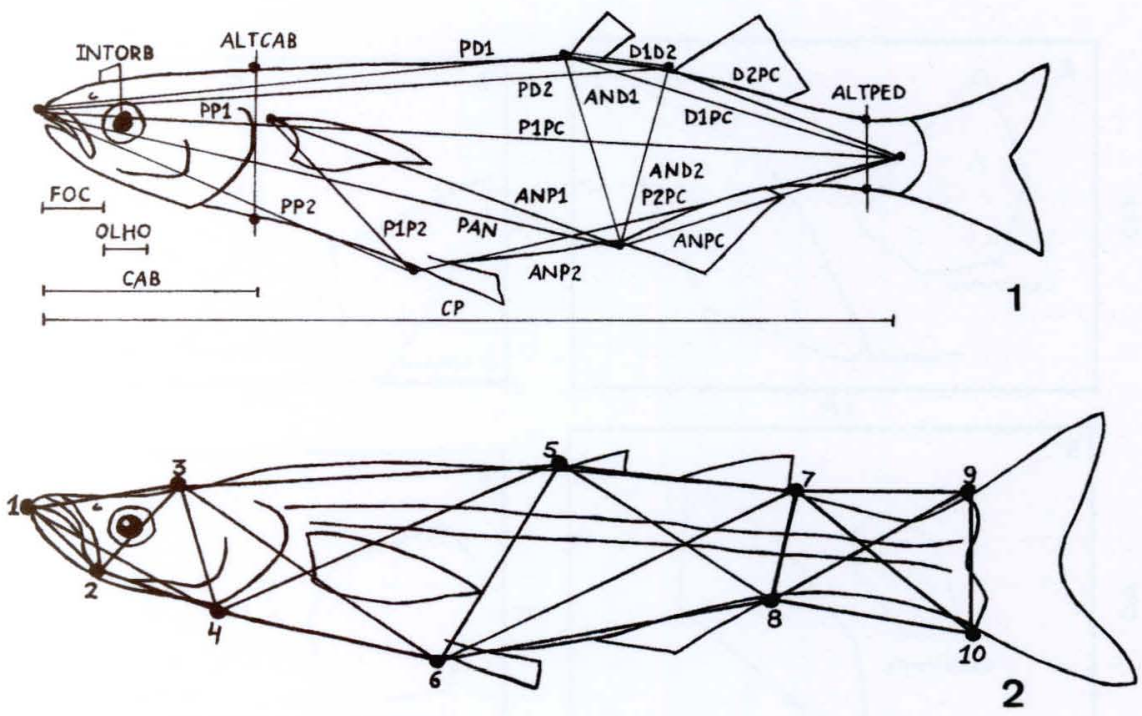

Figs 1-2. (1) Diagrama de medidas tradicionais em Odontesthes humensis: seleção das principais variáveis morfométricas (descrição no texto); (2) Diagrama da rede de treliça para Odontesthes bonariensis, obtida a partir dos marcos anatômicos numerados (descrição no texto).

As medidas foram transformadas em logaritmos decimais a fim de normalizar a distribuição e homogeneizar as variâncias dos dados (SOKAL \& ROHLF 1981; BOOKSTEIN et al. 1985). A comparação entre as séries de medidas, tradicionais e redes de treliça, foi feita pela análise dos componentes principais (denonimados $\mathrm{cp} 1$ e cp2), com a matriz de variância-covariância, para cada uma das séries em separado.

Devido à influência das diferentes etapas de crescimento das espécies amostradas, o conjunto de dados foi ajustado para remover o efeito alométrico, observado no eixo 1, através do método de projeções ortogonais (BURNABY 1966), sendo a análise dos componentes principais denominada CP1 e CP2.

O processamento dos dados foi efetuado através do programa NTSYS-pc (ROHLF 1998).

\section{RESULTADOS}

A análise dos componentes principais para as duas séries de variáveis morfométricas, tradicionais e com redes de treliça, resultou na formação de gráficos de dispersão dos pontos, onde foram observados dois grupos, indicando que a diferenciação específica entre as espécies $O$. bonariensis e $O$. humensis foi devida à variação morfológica (Fig. 3).

$\mathrm{Na}$ análise com variáveis tradicionais os dois primeiros componentes principais explicaram cerca de $93,2 \%$ (cp1) e 2,1\% (cp2) da variação total, enquanto na análise com redes de treliça foram $89,2 \%$ (cp1) e 3,6\% (cp2) da variação total. 

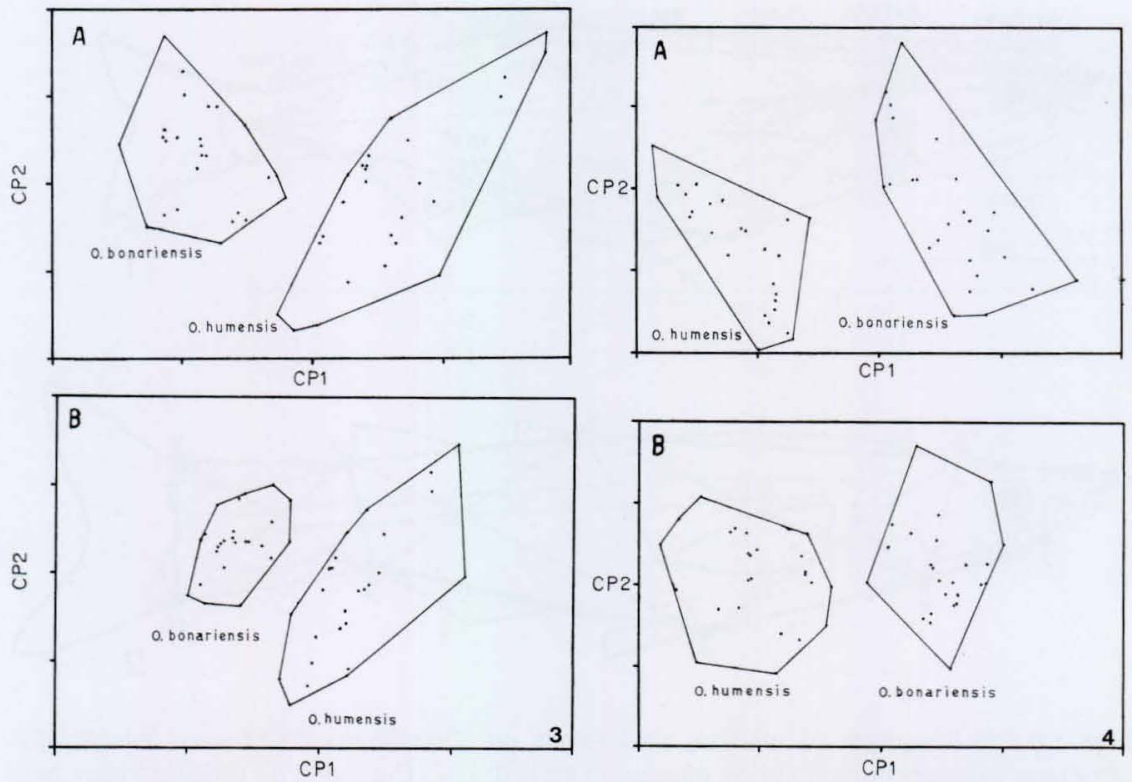

Figs 3-4. Análise dos componentes principais (3) e dos componentes principais ajustada pelas projeções ortogonais de Burnaby (4) realizada com 26 exemplares de Odontesthes bonariensis e 25 exemplares de $O$. humensis. (A) medidas tradicionais (B) medidas com rede de treliça.

O primeiro componente principal, nas duas séries, apresentou coeficientes com valores aproximados e sinal positivo, sendo interpretado como fator tamanho, resultante das diferentes etapas de crescimento dos peixes. O segundo componente apresentou coeficientes positivos e negativos, com diferentes valores, sendo interpretado como mudanças na forma dos organismos. As variáveis cujos coeficientes resultaram positivos, estiveram relacionadas com a região anterior do corpo, enquanto as com coeficientes negativos relacionaram-se com a região do pedúnculo caudal (Tab. I).

Como os grupos não se separam totalmente pelo eixo do componente 2, que representa a forma dos organismos, o conjunto de dados foi então ajustado para remover o efeito do fator tamanho, através do método de projeções ortogonais. O resultado deste ajuste demonstrou, claramente, que as espécies se diferenciaram também através da forma (Fig. 4). Nesta análise, os dois primeiros componentes principais ajustados, explicaram cerca de $47,7 \%$ (CP1) e 13,1\% (CP2) da variação entre grupos, para as variáveis tradicionais e, $54,2 \%(\mathrm{CP} 1)$ e $10,7 \%(\mathrm{CP} 2)$ para as redes de treliça.

Nas duas séries de medidas, o primeiro componente apresentou variáveis com coeficientes positivos e negativos, sendo interpretado como mudanças na forma dos organismos. As variáveis cujos coeficientes resultaram positivos, estiveram relacionadas com a região anterior do corpo, enquanto as com coeficientes negativos relacionaram-se com a região do pedúnculo caudal (Tab. II). 
Tabela I. Análise dos componentes principais para 26 exemplares de Odontesthes bonariensis e 25 de 0 . humensis. Caracteres morfométricos transformados em logaritmos, coeficientes obtidos com dados não ajustados para as medidas tradicionais e redes de treliça. Variáveis encontram-se descritas no texto.

\begin{tabular}{|c|c|c|c|c|c|}
\hline \multirow{2}{*}{ Variáveis } & \multicolumn{2}{|c|}{ Medidas tradicionais } & \multicolumn{3}{|c|}{ Medidas com redes de treliça } \\
\hline & CP1 & $\mathrm{CP2}$ & & CP1 & CP2 \\
\hline $\mathrm{pd} 2$ & 0.191 & 0,054 & $1-3$ & 0,218 & $-0,005$ \\
\hline pd1 & 0,217 & 0,147 & $1-2$ & 0,280 & 0,320 \\
\hline Pan & 0,204 & 0,026 & $1-4$ & 0,267 & 0,277 \\
\hline pp2 & 0,230 & 0,128 & $2-3$ & 0,224 & 0,024 \\
\hline pp1 & 0,238 & 0,090 & $2-4$ & 0,245 & 0,320 \\
\hline $\mathrm{Cab}$ & 0,241 & 0.168 & $3-4$ & 0,222 & 0,027 \\
\hline Altcab & 0,242 & 0,162 & $3-5$ & 0,237 & 0,036 \\
\hline Foc & 0,259 & 0,218 & $3-6$ & 0,238 & 0,080 \\
\hline Olho & 0,091 & 0,018 & $4-5$ & 0,223 & $-0,006$ \\
\hline Intorb & 0.157 & $-0,046$ & $4-6$ & 0,242 & 0,028 \\
\hline Boca & 0,264 & 0,184 & $5-6$ & 0,201 & $-0,116$ \\
\hline Max & 0,274 & 0,248 & $5-7$ & 0,223 & 0,063 \\
\hline Altped & 0,203 & $-0,097$ & $5-8$ & 0,209 & 0,019 \\
\hline p1-p2 & 0,209 & 0,130 & $6-7$ & 0,206 & $-0,053$ \\
\hline p1pC & 0,187 & $-0,215$ & $6-8$ & 0,197 & $-0,033$ \\
\hline p2pc & 0,170 & $-0,201$ & $7-8$ & 0,200 & $-0,090$ \\
\hline an-pc & 0,167 & $-0,196$ & $7-9$ & 0,124 & $-0,447$ \\
\hline $\mathrm{d} 1 \mathrm{pc}$ & 0,155 & $-0,433$ & $7-10$ & 0,131 & $-0,376$ \\
\hline d2pc & 0,148 & $-0,588$ & $8-9$ & 0,168 & $-0,309$ \\
\hline d1-d2 & 0,174 & $-0,136$ & $8-10$ & 0,129 & $-0,457$ \\
\hline an-d1 & 0,195 & $-0,157$ & $9-10$ & 0,208 & $-0,069$ \\
\hline an-d2 & 0,190 & $-0,147$ & & & \\
\hline an-p1 & 0,198 & $-0,013$ & & & \\
\hline Variância (\%) & 93,2 & 2,1 & & 89,2 & 3,6 \\
\hline
\end{tabular}

Tabela II. Análise dos componentes principais para 26 exemplares de Odontesthes bonariensis e 25 O. humensis. Caracteres morfométricos transformados em logaritmos, coeficientes obtidos com dados ajustados para o efeito tamanho para as medidas tradicionais e redes de treliça. Variáveis encontram-se descritas no texto.

\begin{tabular}{|c|c|c|c|c|c|}
\hline \multirow{2}{*}{ Variáveis } & \multicolumn{2}{|c|}{ Medidas tradicionais } & \multicolumn{3}{|c|}{ Medidas com redes de treliça } \\
\hline & $\mathrm{CP} 1$ & $\mathrm{CP} 2$ & & $\mathrm{CP} 1$ & $\mathrm{CP} 2$ \\
\hline $\mathrm{pd} 2$ & 0.105 & 0,023 & $1-3$ & 0,034 & $-0,157$ \\
\hline $\mathrm{pd} 1$ & 0,125 & $-0,030$ & $1-2$ & 0,337 & $-0,663$ \\
\hline Pan & 0,116 & 0,114 & $1-4$ & 0,355 & 0,101 \\
\hline pp2 & 0,189 & 0,010 & $2-3$ & 0,055 & $-0,094$ \\
\hline pp1 & 0,142 & $-0,004$ & $2-4$ & 0,422 & 0,666 \\
\hline $\mathrm{Cab}$ & 0,256 & 0,033 & $3-4$ & 0,018 & 0,013 \\
\hline Altcab & 0,243 & 0,048 & 3-5 & 0,101 & $-0,011$ \\
\hline Foc & 0,279 & $-0,026$ & $3-6$ & 0,165 & 0,071 \\
\hline Olho & $-0,142$ & $-0,355$ & $4-5$ & 0,056 & $-0,014$ \\
\hline Intorb & $-0,367$ & $-0,640$ & $4-6$ & 0,125 & 0,032 \\
\hline Boca & 0,255 & $-0,023$ & $5-6$ & $-0,100$ & $-0,077$ \\
\hline $\operatorname{Max}$ & 0,329 & 0,021 & $5-7$ & 0,104 & 0,056 \\
\hline Altped & $-0,030$ & 0,034 & $5-8$ & 0,048 & 0,013 \\
\hline p1-p2 & 0,057 & $-0,218$ & $6-7$ & $-0,017$ & 0,006 \\
\hline $\mathrm{p} 1 \mathrm{pC}$ & $-0,128$ & 0,168 & $6-8$ & $-0,003$ & 0,032 \\
\hline p2pc & $-0,146$ & 0,095 & $7-8$ & $-0,143$ & $-0,066$ \\
\hline an-pc & $-0,238$ & $-0,063$ & $7-9$ & $-0,376$ & 0,188 \\
\hline d1pc & $-0,307$ & 0,294 & $7 \cdot 10$ & $-0,336$ & 0,025 \\
\hline $\mathrm{d} 2 \mathrm{pc}$ & $-0,363$ & 0,514 & $8-9$ & $-0,240$ & 0.080 \\
\hline $\mathrm{d} 1-\mathrm{d} 2$ & $-0,106$ & 0,045 & $8-10$ & $-0,398$ & 0,063 \\
\hline an-d1 & $-0,138$ & 0,018 & $9-10$ & 0,008 & 0,068 \\
\hline an-d2 & $-0,139$ & $-0,035$ & & & \\
\hline an-p1 & 0,011 & $-0,005$ & & & \\
\hline Variância (\%) & 47,7 & 13,1 & & 54,2 & 10,8 \\
\hline
\end{tabular}




\section{DISCUSSÃO}

O uso de caracteres morfométricos tradicionais está relacionado com a obtenção de medidas longitudinais ao eixo do corpo, armazenando grande quantidade de informações repetitivas em uma mesma região, enquanto os caracteres na direção oblíqua estão ausentes (Fig. 1). As medidas concentram-se em uma mesma região, produzindo uma cobertura desigual da forma do corpo. A medição tradicional reúne uma mescla de informações entre as medidas extremas e as anatômicas. As extremas são distâncias máximas e mínimas, que podem não ser homólogas de um indivíduo a outro, enquanto as anatômicas são identificadas como medidas verdadeiras, unindo pontos homólogos (BOOKSTEIN et al. 1985). Outra combinação de informações é a utilização de medidas muito longas, cruzando várias unidades de crescimento (ex: ossos) e outras curtas, contendo informações localizadas, porém mais úteis (Humphries et al. 1981). Por isso o uso de caracteres morfométricos tradicionais pode dificultar a interpretação dos resultados quando utilizados na diferenciação de formas.

STRAUSS \& BOOKSTEIN (1982) apresentaram um método para obter a estrutura da forma dos organismos, tal qual uma rede de distâncias medidas (truss network). Na figura 2, observa-se que as distâncias medidas cobrem por igual a forma do peixe, incluindo aquelas na direção oblíqua, sendo todas originadas de pontos anatômicos homólogos entre os exemplares.

Uma das aplicações da morfometria multivariada em relação à diferença de formas, é o uso da técnica de componentes principais, estimada a partir de fatores, representando tamanho e forma. Nesta técnica são utilizadas medições lineares e álgebra de matrizes, envolvendo a extração de autovalores e autovetores de matrizes de covariância (NEFF \& MARCUS 1980; REIS 1988).

O procedimento na análise dos componentes principais tem sido interpretar o primeiro componente (cp1), como uma medida de variação de "tamanho" por apresentar coeficientes com valores aproximados e sinais positivos, enquanto que o segundo eixo (cp2) e demais, são interpretados como mudanças na forma dos organismos, quando tiverem coeficientes com diferentes sinais e valores (JOLICOEUR 1963; HUMPHRIES et al. 1981; BOOKSTEIN et al. 1985; REIS 1988). O tamanho é estimado pelo primeiro autovetor de uma matriz de covariância dos caracteres, cuja medição foi transformada para logaritmo (REIS 1988).

No estudo comparativo das diferenças de forma entre duas espécies é interessante separar as informações obtidas com o tamanho, em função do crescimento diferenciado dos organismo (HUMPHRIES et al. 1981; BOOKSTEIN et al. 1985; REIS et al. 1987). Através do gráfico de dispersão da figura 3 foram observadas duas nuvens de pontos. Devido à influência das diferentes etapas de crescimento das espécies amostradas, a separação pelo eixo 2 , que representa a forma dos peixes, não foi totalmente identificada.

$\mathrm{Na}$ análise com dados ajustados pelas projeções ortogonais de BURNABY (1966), as informações de tamanho são computadas e separadas das informações de forma. A nova matriz resultará em uma associação de comprimento e largura, conforme os sinais (+) e (-) apresentados pelos coeficientes de CP1 e CP2. Os 
coeficientes indicaram um contraste de variáveis, cujos sinais opostos $(-/+)$ representam a noção de forma no contexto da morfometria convencional (MONTEIRO \& REIS 1998). A figura 4 mostrou a utilização do conjunto de dados ajustados, no qual foi possível reconhecer diferenças na forma, indicando distintas unidades específicas.

É interessante observar que as variáveis com maiores coeficientes, positivos e negativos, foram as mesmas na análise ajustada e sem ajuste, por isso as diversas medidas obtidas na parte anterior do corpo resultaram na discriminação das duas espécies.

Estudos sobre a morfometria multivariada em peixes-rei, a partir de medidas tradicionais, tem demonstrado bons resultados na diferenciação morfométrica (BEMVENUTI 1997, 2000). No presente trabalho, os autores se preocuparam em demonstrar que as medidas com redes de treliça, resultaram numa melhor diferenciação entre os grupos de $O$. bonariensis e $O$. humensis, uma vez que o método aperfeiçoa a discriminação pela forma com o uso de medidas cruzadas, não consideradas no método tradicional (HuMPHRIES et al. 1981). O padrão de diferenciação entre os grupos foi também observado pela posição dos pontos no gráfico, situados mais próximos uns dos outros na rede de treliça do que nas medidas tradicionais (Fig. 4).

AGRADECIMENTOS. Os autores agradecem ao pescador Waldeci Peres (Taim, Rio Grande, Rio Grande do Sul) pelo auxílio nas coletas, ao acadêmico Eduardo Wenzel Brião pela colaboração no tratamento estatístico e ao CNPq as bolsas de Iniciação Científica.

\section{REFERÊNCIAS BIBLIOGRÁFICAS}

Bemvenuti, M.A. 1997. Relações morfológicas e osteológicas entre Odontesthes perugiae e $O$. mirinensis (Teleostei: Atherinidae, Atherinopsinae) Atlântica, Rio Grande, 19: 113-131.

- 2000. Diferenciação geográfica do peixe-rei Odontesthes argentinensis (Atherinopsidae), no extremo sul do Brasil, através da morfometria multivariada. Atlântica, Rio Grande, 22: 71-79

- 2002. Diferenciação morfológica das espécies de peixes-rei, Odontesthes Evermann \& Kendall (Osteichthyes, Atherinopsidae) no extremo sul do Brasil: morfometria multivariada. Revta bras. Zool. 19 (1): 251-287.

Bookstein, F.L 1993. A brief history of the morphometric synthesis, p. 15-40. In: L.F. Marcus; E. Bello \& A. Garcia-Valdecasas (Eds). Contributions to morphometrics. Monografias del Museo Nacional de Ciencias Naturales 8, Madri.

Bookstein, F.L.; B. ChernofF; R. Elder; J. Humphries; G. Smith \& R. StRauss. 1985. Morphometrics in evolutionary biology. Acad. Nat. Sci. Philad. (15): 1-277.

BuRnABY, T.P. 1966. Growth invariant discriminant functions and generalized distances. Biometrics 22: $96-110$.

Humphries, J.M.; F.L. Bookstein; B. ChernofF; G.R. Smith; R.L. Elder \& S.G. Poss. 1981. Multivariate discrimination by shape in relation to size. Syst. Zool. 30 (3): 291-308.

JoLicoeur, P. 1963. The multivariate generalization of the allometry equation. Biometrics 19: 497-499.

Monteiro, L.R. \& S.F. Reis. 1998. Princípios de Morfometria Geométrica. Ribeirão Preto, Ed. Holos, $188 \mathrm{p}$.

NEFF, N.A. \& L.F. MARCUS. 1980. A survey of multivariate methods for systematics. New York Privately published, 234p.

REIS, S.F. 1988. Morfometria e estatistica multivariada em biologia evolutiva. Revta bras. Zool. 5 (4): 571-580. 
Reis, S.F.; R.A. Cunha; J.C. Garavello \& A.S. Abe. 1987. Discriminação pela forma em relação ao tamanho: um exemplo com peixes do gênero Leporinus. Ci. Cult. 39 (8): 757-761.

REYMENT, R.A. 1985. Multivariate morphometrics and analysis of shape. Mathematical Geology 17 (6): 591-609.

Reyment, R.A.; R.E. Blackith; N.A. CAMPBell. 1984. Multivariate morphometrics. London, Academic Press, $2^{\text {nd }}$ ed., 233p.

RoHLF, F.J. 1990. Morphometrics. Ann. Rev. Ecol. Syst. 21: 299-316.

1998. NTSYS-pc. Numerical taxonomy and multivariate analysis system. New York, Department of Ecology and Evolution, Stony Brook, Version 2.0.

SOKAL, R.R. \& F.J. RoHLF. 1981. Biometry. San Francisco, W.H. Freeman, $2^{\text {nd }}$ ed., 859p.

StRauss, R.E. \& F.L. Bookstein. 1982. The truss: body form reconstructions in morphometrics. Syst. Zool. 31 (2): 113-135.

Recebido em 24.VIII.2001; aceito em 27.VIII.2002. 